\title{
Case Files of the University of California San Francisco Medical Toxicology Fellowship: Lamotrigine Toxicity
}

\author{
Michelle Fleurat • Craig Smollin
}

Published online: 4 February 2012

(C) American College of Medical Toxicology 2012

Keywords Lamotrigine $\cdot$ Seizure $\cdot$ Movement disorders

\section{Case Presentation}

A 56-year-old man was brought to the emergency department (ED) after he was found seizing on the floor by his family. His brother described hearing a thump in his room and witnessed him having a full body clonic seizure that lasted approximately $2 \mathrm{~min}$. When emergency medical service arrived, he was found on the floor with altered mental status and with signs of facial trauma. No medications were administered en route to the hospital.

On arrival to the ED, he was initially confused and mumbling incoherently. He was noted to be only oriented to self, and would not reliably follow commands. Approximately $1 \mathrm{~h}$ after arrival, he became agitated, seemed more confused, and would not follow any commands. Initial vital signs were: blood pressure, 147/76 $\mathrm{mmHg}$; pulse, 81/min; respiratory rate, $18 / \mathrm{min}$; temperature, $35^{\circ} \mathrm{C}$ by tympanic measurement; and oxygen saturation was $100 \%$ on room air. On physical exam, his pupils were $4 \mathrm{~mm}$ and reactive bilaterally. Extraocular movements appeared intact by observation only and there was no nystagmus. He had a nasal

M. Fleurat $\cdot$ C. Smollin

University of California, San Francisco,

Medical Toxicology Fellowship,

San Francisco, CA, USA

\section{Fleurat $(\square)$}

California Poison Control System, San Francisco Division,

University of California, San Francisco,

Box 1369, San Francisco, CA 94143-1369, USA

e-mail: mfleur77@gmail.com deformity and laceration to the right side of his tongue. The chest exam was normal with the exception of a large median sternotomy scar. His abdomen was soft, nontender, and there were no signs of bladder distension. Bowel sounds were noted to be present. There was no evidence of bowel or bladder incontinence. The neurological exam was notable for decreased attention, inability to follow commands, inability to repeat examiner's phrases, and markedly reduced language skills. The patient had spastic muscle tone with resistance to motion both with flexion and extension of all extremities. Deep tendon reflexes were noted to be $2+$ in bilateral upper extremities and $3+$ in bilateral lower extremities without clonus.

\section{How Can We Classify This Patient's Neurologic Findings?}

The brother's description of events suggests a generalized tonic-clonic seizure. The patient was witnessed to have full body "shaking" movements lasting approximately $2 \mathrm{~min}$. Evidence of tongue trauma also suggests seizure activity. His altered mental status on arrival in the ED is consistent with a post-ictal state. The differential diagnosis for seizure is broad and is considered in detail below. However, the patient's initial neurologic exam, particularly the description of his tone and reflexes, raises the possibility of alternative or additional explanations. Disorders of movement, with or without altered mental status, may mimic seizure activity and should be considered in this case.

While the pathophysiology of movement disorders is complex, in general they are caused by dysfunction within the basal ganglia. It can be useful to divide disorders of movement into those causing increased and involuntary 
movements (hyperkinetic disorders) and those causing decreased movements or rigidity (hypokinetic disorders). Hyperkinetic movement disorders include myoclonus, chorea, and dystonia. Myoclonus consists of sudden, brief shocklike movements that may be due to muscle contractions or loss of muscle tone. For example, myoclonic encephalopathy can be seen with carisoprodol overdose. Chorea consists of involuntary, irregular, purposeless movements that "flow" into one another in a random fashion. Chorea can be caused by hereditary conditions, as in Huntington's chorea, or acquired, as in the dance-like movements sometimes seen after exposure to cocaine. Dystonia describes involuntary, sustained muscle contractions that produce twisting or squeezing movements and perhaps are more accurately described as postures [1]. The term athetosis refers to slow, more writhing movements, but is sometimes used interchangeably with dystonia. Acute dystonic posturing, such as torticollis or trismus, may result from use of dopamine antagonist medications. The risk is greatest with antipsychotics that have little anticholinergic effect such as piperazine phenothiazines, butyrophenones, and thioxanthines [2]. Dystonic reactions generally occur within 1 week of starting the drug and often within the first 24-48 h [3]. While there are many possible etiologies of myoclonus, chorea, and dystonia, Table 1 summarizes some of the toxins and drugs that may be responsible. Of note, there is a significant degree of overlap among the drugs and toxins that can result in these various disorders and a single drug or toxin may produce a variety of hyperkinetic movement disorders.

Hypokinetic movement disorders include neuroleptic malignant syndrome and acute parkinsonism among others. Parkinsonian movements may be organic in nature or drug related. The 4- to $6-\mathrm{Hz}$ tremor of parkinsonism is typically most obvious at rest and can increase at times of emotional stress and often improves during voluntary activity. Both dopamine and acetylcholine are present in the corpus striatum, where they act as neurotransmitters. It is generally believed that the dopamine depletion disturbs the normal balance between these two antagonistic neurotransmitters. The disturbance in tone is responsible for the flexed posture of many patients with parkinsonism. The most disabling feature of this disorder is hypokinesia, a slowness of voluntary movement and a reduction in automatic movement, such as swinging the arms while walking [2]. Drug-induced parkinsonism, like dystonia, is most often seen with dopamine antagonists, however the symptoms tend to develop several days after starting drug therapy [3]. Young men are most susceptible to dystonic reactions, while elderly men and women tend to be more susceptible to parkinsonian reactions to antipsychotics $[4,5]$.

Neuroleptic malignant syndrome (NMS) is a rare complication of treatment with antipsychotic drugs and is manifested by rigidity, fever, altered mental status, and
Table 1 Selected toxins and drugs causing hyperkinetic movement disorders

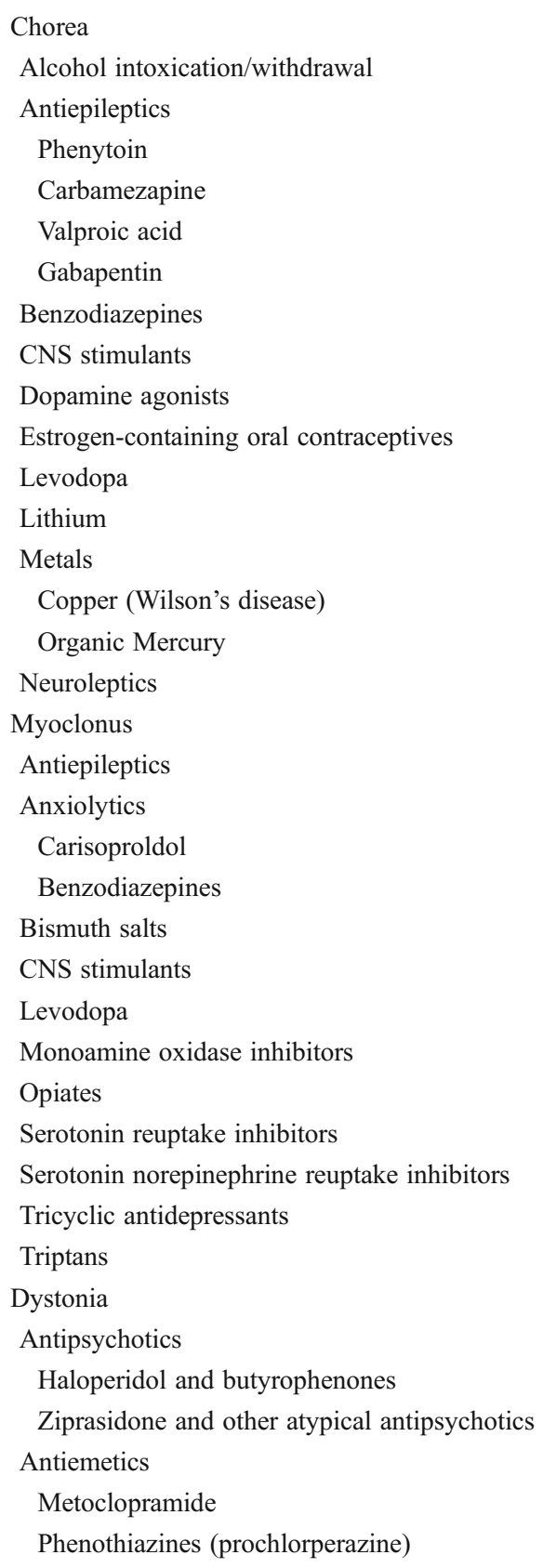

Compiled/adapted from: Ref. \#1; Pract Neurol 2007;7(6):360-373; J Neurol Neurosurg Psychiatr 1998;65(4):436-445; CNS Drugs 2004:18 (2):93-104; Goldfrank's Toxicologic Emergencies, 8th ed, New York: McGraw-Hill Medical, 2006; Poisoning \& Drug Overdose, 5th ed, New York: McGraw-Hill Medical, 2006

autonomic dysfunction. Haloperidol is implicated most often, but NMS can be seen with any antipsychotic drug. Symptoms typically develop over 1-3 days and can occur at any time during the course of treatment. The differential diagnosis includes infection, which must be excluded in any febrile patient [2]. 
Serotonin syndrome is sometimes discussed in the context of hypokinetic movement disorders. Serotonin syndrome results from excessive stimulation of postsynaptic serotonin receptors, either from therapeutic serotonergic drug use, overdose or from interactions of multiple serotonergic drugs or concurrent use of serotonergic drugs and monoamine oxidase inhibitors. Signs and symptoms range from tremor, diaphoresis, restlessness, and diarrhea in mild cases to confusion, hyperthermia, tachycardia, hypertension, and hyperreflexia. The clonus and hyperreflexia are generally more prominent in the lower extremities and clinicians should be aware that muscle rigidity may mask these characteristic signs [6]. Serotonin syndrome occurs in approximately $14-16 \%$ of persons who overdose on selective serotonin reuptake inhibitors (SSRIs) [7].

This patient presented with altered mental status and was described to have "spastic tone" with "resistance to motion" on flexion and extension of all extremities. He did not appear to have tremor, myoclonus, or chorea. In addition to seizures, it is possible that the patient was exhibiting signs of a movement disorder, including NMS, serotonin syndrome, or acute parkinsonism. Further history was obtained from the patient's family, focusing on the past medical history, current medications, as well as history of drug and alcohol abuse.

\section{Case Continuation}

Additional history obtained from the patient's brother revealed that the patient had a history of right frontal lobe resection secondary to an astrocystoma and a resultant seizure disorder. The patient's known outpatient medication regimen consisted of clonazepam $1 \mathrm{mg}$ PO BID and lamotrigine $200 \mathrm{mg}$ PO TID; however, he only intermittently took his medications and has had numerous breakthrough seizures. The patient had a pattern of taking more than the prescribed dose of his medications in the beginning of the month and would run out before his next prescription was ready. He was also known to abuse alcohol.

\section{How Should the Toxicologist Approach the Patient with Seizures?}

Given this additional history, seizure appeared to be the most likely cause of the patient's clinical presentation. The initial management of the patient presenting with a seizure focuses on supportive care measures. These include maintaining a patent airway, ensuring adequate ventilation, and obtaining appropriate intravenous access. Once the ABCs have been addressed, a broad differential diagnosis that includes both nontoxic and toxic etiologies can be formulated. Metabolic derangements, particularly hypoglycemia, should be addressed rapidly. Other nontoxicologic etiologies to consider include trauma, hypoxia, hyperthermia, and infection.

When drugs or toxins are suspected as the potential cause of seizure, there are numerous agents to consider. Categories include analgesics, anticonvulsants, cellular asphyxiants, drugs of abuse, envenomations, heavy metals, plants, herbs and natural products, psychiatric medications, and rodenticides among others (see Table 2). While there may be clues in the clinical history that suggest the causative agent, the toxicologist should seek collateral information from paramedics, family members, and perhaps even the patient's dispensing pharmacy. For example, a sympathomimetic toxidrome preceding seizure activity may suggest cocaine or amphetamines. A patient with a history of foraging for mushrooms may have inadvertently ingested a species of Gyromitra. A patient with a psychiatric history may have overdosed on an SSRI or tricyclic antidepressant, while a patient with a history of treatment for tuberculosis may have overdosed on isoniazid. The social history may suggest withdrawal from ethanol or other sedative-hypnotic agents.

The differential diagnosis for this patient included post-traumatic versus other intracranial abnormalities, sedative-hypnotic withdrawal, as well as a subtherapeutic lamotrigine level resulting in breakthrough seizures. It is important to also consider the possibility of lamotrigine overdose in this case.

A seemingly contradictory cause of seizures is the ability of anticonvulsants in overdose to induce convulsions. This is a well-recognized complication, although the mechanism is poorly understood. There is very little experimental evidence and most theories are speculative and unproven [8]. One theory is that the anticonvulsant in high concentrations may have a depressant effect on inhibitory interneurons resulting in disinhibition of excitatory neurons and facilitation of epileptic discharges [9]. Carbamazapine remains the most commonly cited anticonvulsant to cause seizures in overdose, with one case series suggesting that the 10,11-epoxide may be responsible [10]. Another suggested mechanism is that some seizures are precipitated by drowsiness and the sedation produced by anticonvulsant overdose may encourage those susceptible seizure disorders [8].

\section{Case Continuation}

After an hour in the ED, the patient had increased agitation and trouble following commands. He was placed in soft restraints and required multiple doses of lorazepam for sedation. A Foley catheter was inserted, with an initial urine output of $1,300 \mathrm{~cm}^{3}$. He was not noted to have other anticholinergic signs including mydriasis, dry skin, or tachycardia. 
deviated nasal septum, and evidence of a prior frontoparietal craniotomy with significant underlying encephalomalacia.

The emergency physician contacted the Poison Control Center and the patient was admitted to the Medicine service. He was first seen by the toxicology service on hospital day 2 and on evaluation had persistent altered mental status. $\mathrm{He}$ was oriented to person and place only and was slow to process and answer questions. He easily lost attention and required questions repeated to him on several occasions. Physical exam was notable for: pupils $3 \mathrm{~mm}$ bilaterally and reactive, increased muscle tone more prominent in the lower extremities without clonus, patellar reflexes $3+$ bilaterally and brisk.

The initial thought of the admitting team was that the patient most likely suffered a breakthrough seizure due to poor medication compliance. Rhabdomyolysis was thought to be secondary to persistent rigidity, spasticity, and agitation in addition to initial seizure activity. The consulting neurologist agreed with this assessment. They attributed his persistent altered mental status to a prolonged postictal state. In fact, previous records detailed a similar admission 18 months earlier, which described a generalized tonicclonic seizure and a prolonged postictal state. No lamotrigine level was sent at that time. Neurology recommended restarting the patient's lamotrigine. The toxicology service was concerned that the patient's seizure and persistent altered mental status might represent lamotrigine toxicity.

\section{Describe the Mechanism of Action and Pharmacokinetics of Lamotrigine?}

Lamotrigine is a phenyltriazene agent that diminishes excitatory neurotransmitter release, primarily glutamate, by inhibiting voltage-gated sodium channel opening. It binds to the channel pore in the inactive open state and prevents recovery. The mechanism is similar to that of carbamazepine and phenytoin, but lamotrigine is effective for a greater spectrum of seizure disorders, suggesting that it may have additional actions [11]. Both simple and complex partial seizures and secondarily generalized tonic-clonic seizures are reduced by lamotrigine. Generalized seizures, particularly absence seizures, atonic seizures, and Lennox-Gastaut syndrome, a severe form of childhood epilepsy, tend to be more responsive to lamotrigine than partial seizures [12]. At therapeutic concentrations, lamotrgine activity is selective for high-frequency firing. At toxic concentrations, both high-frequency firing and spontaneous sodium channel activity are inhibited [13]. Lamotrigine is also effective in the treatment of mood disorders such as bipolar disorder through the inhibition of serotonin reuptake. While further studies are needed, it appears to be most effective for patients in whom depressive symptoms predominate over mania [14].

Lamotrigine is completely absorbed from the gastrointestinal tract with minimal first-pass metabolism; bioavailability is $98 \%$. It is metabolized almost entirely by glucuronidation, producing inactive metabolites that mainly consist of lamotrigine- $2 N$-glucuronide, and to a lesser extent the $5 N$-glucuronide, $N$-oxide, and $N$-methyl metabolites, all of which are renally excreted [15]. Lamotrigine is not extensively protein bound $(50-55 \%)$ and the volume of distribution is approximately $1.2 \mathrm{~L} / \mathrm{kg}$. Peak plasma concentrations are reached in approximately $1-3 \mathrm{~h}$ at therapeutic dosing, with a half-life of 24.1-37.4 h.

\section{What is the Typical Course of Lamotrigine Overdose? How Common are Lamotrigine-Induced Seizures?}

A retrospective review of poison center data regarding 493 cases involving single substance lamotrigine exposures showed that the majority of patients $(52.1 \%)$ exposed to lamotrigine in overdose experienced no toxic clinical effects. The most common clinical effects reported in overdose were drowsiness/lethargy (20.9\%), vomiting (11\%), nausea (5.1\%), ataxia $(4.9 \%)$, dizziness/vertigo (4.5\%), and tachycardia $(4.3 \%)$. Major clinical effects included coma $(n=6)$, seizures $(n=8)$, and respiratory depression $(n=3)$. Medical outcome was reported as minor in $150(30.4 \%)$, moderate in 73 $(14.8 \%)$, and major in 13 (2.6\%) cases. There were no deaths [16]. The incidence of serious rash in the case of therapeutic dosing in pediatric patients $(\sim 0.8 \%)$ is higher than in the adult population $(0.3 \%)$ [11]. There is no evidence that the incidence of rash is higher in overdose.

Although rare, lamotrigine overdose-induce seizures have been reported several times in the medical literature and should be considered in the assessment of the seizure patient. A 42-year-old woman presented to hospital with generalized seizures after deliberate lamotrigine overdose. Seizure activity was promptly terminated after intravenous benzodiazepine administration, and the patient subsequently made a complete recovery. The serum lamotrigine concentration was $30 \mathrm{mg} / \mathrm{L}$ at $1.3 \mathrm{~h}$ post-ingestion, which is substantially higher than the therapeutic reference range. Levels greater that $14 \mathrm{mg} / \mathrm{L}$ are potentially toxic [13]. The estimated elimination half-life was determined to be $18.3 \mathrm{~h}$ [17]. In another case, a 29-year-old man presented after ingestion of an unknown amount of lamotrigine and ethanol. He proceeded to have several tonic-clonic seizures in the ED that terminated with lorazepam. The ethanol level was $191 \mathrm{mg} / \mathrm{dL}$ and the lamotrigine level was $25.2 \mathrm{mg} / \mathrm{L}$ [18]. A 2-year-old boy ingested $800 \mathrm{mg}$ of lamotrigine, suffered a tonic-clonic seizure followed by tremors, weakness, ataxia, and hypertonia. His serum level returned at $3.8 \mathrm{mg} / \mathrm{L}[19]$. 
How Does the Clinician Differentiate between Seizures Caused by Subtherapeutic versus Supratherapeutic Levels of an Anticonvulsant Drug for which Levels are not Rapidly Available?

Unfortunately, this is a question that challenges toxicologists in all cases involving the newer anticonvulsantslamotrigine included. Drug levels for the new anticonvulsants are "send out" tests and generally cannot be obtained in a clinically useful timeframe. Without quantitative levels, the toxicologist is often at a loss as to how to proceed with the management of the seizing overdose patient. This can lead to contradictory recommendations from different consulting services and general uncertainty in the recommendations that we give. In this case, the consulting neurologists recommended restarting the lamotrigine on hospital day 2 , as their suspected diagnosis at that time was medication noncompliance and low serum lamotrigine levels. While the toxicology service acknowledged that this was a possibility, we suggested withholding lamotrigine until toxicity was ruled out. Lee et al. studied the effect of a toxicology service during a 1-year period and showed a neutral effect on the ED length of stay, but a decrease in the hospital length of stay especially in complicated patients [20]. Another study found that patients seen by the medical toxicology consult service consumed fewer health care resources in the form of less decontamination and fewer laboratory tests [21]. While neither of these studies specifically evaluated cases of anticonvulsant toxicity, they suggest a potential clinical benefit of following toxicology recommendations in cases of known or suspected overdose.

\section{Case Continuation}

A lamotrigine level was sent to the experimental lab associated with the Poison Control Center, and the admitting team elected to discontinue his lamotrigine. Instead he was loaded with phenytoin and started on phenytoin $100 \mathrm{mg}$ IV TID. Over the next $24 \mathrm{~h}$, the patient slowly returned to his baseline mental status. Other than mild rhabdomyolysis (peak $\mathrm{CK}=$ $8,232 \mathrm{U} / \mathrm{L}$ ), the majority of his labs were unremarkable and his BUN and creatinine remained normal. Thyroidstimulating hormone was normal and rapid plasma reagin was nonreactive. His QRS duration decreased to $96 \mathrm{~ms}$ and the QTc decreased to $433 \mathrm{~ms}$ without intervention.

\section{Which Drugs and Conditions when Added to Lamotrigine can Increase Toxicity?}

The metabolism of lamotrigine to glucuronides by the liver (approximately $63 \%$ of an ingested dose) can be quite variable depending on the chronic co-ingestion of hepatic mixed function oxidase system inducers such as phenytoin, phenobarbital, carbamazepine, rifampicin, and St John's wort or the presence of P450 enzyme inhibitors such as valproate. Alteration in metabolism is not expected to occur after single, even large, coingestions of an inducer, but may occur if the inducer has been used as a chronic medication [18]. The serum lamotrigine concentration is increased with valproic acid administration, likely secondary to competition for glucuronidation. This competition may prolong lamotrigine's half-life to $60 \mathrm{~h}$. Sertaline may also result in similar lamotrigine intoxication. The $\mathrm{T}^{1 / 2}$ is $8-20 \mathrm{~h}$ in the presence of concomitant enzyme inducers [22]. With severe hepatic dysfunction the $\mathrm{T}^{1 / 2}$ may be increased to a median of $110 \mathrm{~h}$ and in the case of severe renal dysfunction to $50 \mathrm{~h}$ [23].

\section{Case Conclusion}

In discussion with the inpatient neurology service, as well as the patient's outpatient neurologist, the admitting team determined that the patient's persistent altered mental status and spasticity were related his postictal state in conjunction with his right frontal lobe resection. The patient's mental status improved to the point that he was deemed safe for discharge 4 days after admission, with outpatient neurology follow-up. His physical exam at the time of discharge was notable for baseline mental status, alert and oriented to person, place and time, but the patient continued to exhibit decreased attention. He had normal muscle strength and tone throughout, with $2+$ patellar and biceps reflexes.

The discharge diagnosis was seizure with a prolonged postictal state. The patient was discharged to home with his brother. His clonazepam and lamotrigine were not restarted and he was continued on extended-release phenytoin $300 \mathrm{mg}$ PO daily. His lamotrigine levels returned after the time of discharge at $33 \mu \mathrm{g} / \mathrm{mL}$ on HD 1 and $20 \mu \mathrm{mL}$ on HD 2 (normal therapeutic range is $3-11 \mu \mathrm{mL}$ ), confirming the diagnosis of drug toxicity.

Conflict of interest We have no conflicts to report.

\section{References}

1. Robottom BJ, Factor SA, Weiner WJ (2011) Movement disorders emergencies Part 2: hyperkinetic disorders. Arch Neurol 68 (6):719-724

2. Simon RP, Greenberg DA, Aminoff MJ (2009) Chapter 7. Movement Disorders. In: Aminoff MJ, Greenberg DA, Simon R (eds) 
Clinical neurology, 7th edn. Lange Medical Books/McGraw-Hill, New York, pp 238-269

3. Casey DE (2004) Pathophysiology of antipsychotic drug-induced movement disorders. J Clin Psychiatry 65(Suppl 9):25-28

4. Spina E, Sturiale V, Valvo S et al (1993) Prevalence of acute dystonic reactions associated with neuroleptic treatment with and without anticholinergic prophylaxis. Int Clin Psychopharmacol 8 (1):21-24

5. Gershanik OS (1994) Drug-induced parkinsonism in the aged. Recognition and prevention. Drugs Aging 5(2):127-132

6. Claassen JA, Gelissen HP (2005) The serotonin syndrome. N Engl J Med 352(23):2454-2456

7. Isbister GK, Bowe SJ, Dawson A, Whyte IM (2004) Relative toxicity of selective serotonin reuptake inhibitors (SSRIs) in overdose. J Toxicol Clin Toxicol 42(3):277-285

8. Somerville ER (2006) Seizure aggravation by antiepileptic drugs. Curr Treat Options Neurol 8(4):289-296

9. Perucca E, Gram L, Avanzini G, Dulac O (1998) Antiepileptic drugs as a cause of worsening seizures. Epilepsia 39(1):5-17

10. So EL, Ruggles KH, Cascino GD, Ahmann PA, Weatherford KW (1994) Seizure exacerbation and status epilepticus related to carbamazepine-10,11-epoxide. Ann Neurol 35(6):743-746

11. McNamara JO (2011) Pharmacotherapy of the Epilepsies. In: Brunton LL, Chabner BA, Knollmann BC (eds) Goodman \& Gilman's The Pharmacological Basis of Therapeutics, 12th edn. McGraw-Hill, New York, pp 583-607

12. Fitton A, Goa KL (1995) Lamotrigine. An update of its pharmacology and therapeutic use in epilepsy. Drugs 50(4):691-713
13. Doyon S (2011) Anticonvulsants. In: Nelson LS, Lewin NA, Howland MA et al (eds) Goldfrank's Toxicologic Emergencies, 9th edn. McGraw-Hill Medical, New York, pp 698-710

14. Bourin M, Masse F, Hascoët M (2005) Evidence for the activity of lamotrigine at $5-\mathrm{HT}_{1 \mathrm{~A}}$ receptors in the mouse forced swimming test. J Psychiatry Neurosci 30(4):275-282

15. Ng F, Hallam K, Lucas N, Berk M (2007) The role of lamotrigine in the management of bipolar disorder. Neuropsychiatr Dis Treat 3 (4):463-474

16. Lofton AL, Klein-Schwartz W (2004) Evaluation of lamotrigine toxicity reported to poison centers. Ann Pharmacother 38(11):1811-1815

17. Waring WS. Lamotrigine overdose associated with generalised seizures. BMJ Case Rep 2009, doi:10.1136/bcr.07.2008.0489, Published online 2009 February 16.

18. Schwartz MD, Geller RJ (2007) Seizures and altered mental status after lamotrigine overdose. Ther Drug Monit 29(6):843-844

19. Briassoulis G, Kalabalikis P, Tamiolaki M, Hatzis T (1998) Lamotrigine childhood overdose. Pediatr Neurol 19(3):239-242

20. Lee V, Kerr JF, Braitberg G et al (2001) Impact of a toxicology service on a metropolitan teaching hospital. Emerg Med (Fremantle) 13(1):37-42

21. Clark RF, Williams SR, Nordt SP, Pearigen PD, Deutsch R (1998) Resource-use analysis of a medical toxicology consultation service. Ann Emerg Med 31(6):705-709

22. Chan V, Morris RG, Ilett KF, Tett SE (2001) Population pharmacokinetics of lamotrigine. Ther Drug Monit 23(6):630-635

23. Johannessen SI, Tomson T (2006) Pharmacokinetic variability of newer antiepileptic drugs: when is monitoring needed? Clin Pharmacokinet 45(11):1061-1075 